\title{
Dichloroacetate stimulates changes in the mitochondrial network morphology via partial mitophagy in human SH-SY5Y neuroblastoma cells
}

\author{
DAVID PAJUELO-REGUERA, LUKÁŠ ALÁN, TOMÁŠ OLEJÁR and PETR JEŽEK \\ Department of Membrane Transport Biophysics, No. 75, Institute of Physiology, \\ Academy of Sciences of the Czech Republic, Prague, Czech Republic
}

Received January 29, 2015; Accepted March 11, 2015

DOI: $10.3892 /$ ijo.2015.2953

\begin{abstract}
Dichloroacetate (DCA) is beneficial in cancer therapy because it induces apoptosis and decreases cancer growth in vitro and in vivo without affecting non-cancer cells. DCA stimulates the activity of the enzyme pyruvate dehydrogenase by inhibiting pyruvate dehydrogenase kinase. Consequently, DCA promotes oxidative phosphorylation after glycolysis. Therefore, DCA produces changes in energy metabolism that could affect the mitochondrial network and mitophagy. This investigation determined the effects of DCA treatment on mitophagy in human neuroblastoma SH-SY5Y cells. SH-SY5Y cells were cultured and distributed into 3 groups: control, $\mathrm{NH}_{4} \mathrm{Cl}$ and chloroquine. Each group was treated with DCA at 0, 5, 30 and $60 \mathrm{mM}$ for $16 \mathrm{~h}$. Samples were analyzed for cell viability, mtDNA copy number, mitochondrial network morphology and expression of key proteins involved in mitochondrial dynamics, such as LC3b,
\end{abstract}

Correspondence to: Dr David Pajuelo-Reguera, Department of Membrane Transport Biophysics, No. 75, Institute of Physiology, Academy of Sciences of the Czech Republic, Prague, Czech Republic E-mail: d.pajuel@outlook.com

Abbreviations: 3-MA, 3-methyladenine; ATP, adenosine triphosphate; AU, arbitrary units; BCA, bicinchoninic acid solution; BSA, bovine serum albumin; CHLQ, chloroquine; DCA, dichloroacetate; DMEM, Dulbecco's modified Eagle's medium; DRP1, dynamin related protein 1; FIS1, mitochondrial fission protein 1; LC3b, MAP1LC3B, microtubule-associated protein 1 light chain $3 \beta$; mtDNA, mitochondrial DNA; OPA1, optic atrophy 1; OXPHOS, oxidative phosphorylation; PAGE, polyacrylamide gel electrophoresis; Parkin, E3 ubiquitin-protein ligase parkin; PBS, phosphatebuffered saline; PINK1, PTEN-induced kinase 1; PVDF, polyvinylidene difluoride; RIPA, radioimmunoprecipitation assay buffer; SDS, sodium dodecyl sulfate; TBS, Tris-buffered saline; TBS-T, Tris-buffered saline-Tween; TFAM, transcription factor A, mitochondrial; Tim23, mitochondrial import inner membrane translocase subunit Tim23; TMRE, tetramethylrhodamine ethyl ester

Key words: dichloroacetate, mitochondria, mitophagy, neuroblastoma SH-SY5Y cells, mitochondrial network
FIS1, OPA1, PARKIN and PINK1. In all groups, DCA caused a decrease in cell viability, an induction of autophagy in a dose-dependent manner and a decrease in Tim23, FIS1 and PARKIN protein expression, leading to profound morphological changes in the mitochondrial network resulting in shorter and more fragmented filaments. However, TFAM protein levels remained unchanged. Similarly, the mitochondrial copy number was not significantly different among the treatment groups. In conclusion, DCA induces mitophagy and remodeling of the mitochondrial network. In this remodeling, DCA induces a decrease in the expression of key proteins involved in protein degradation and mitochondrial dynamics but does not significantly affect the mtDNA density. Blocking late phase autophagy increases the effects of DCA, suggesting that autophagy protects the cell, at least partially, against DCA.

\section{Introduction}

Dichloroacetate (DCA) is a metabolic modulator that has been used in humans for decades for the treatment of lactic acidosis and inherited mitochondrial diseases (1). DCA is a pyruvate dehydrogenase kinase (PDK) inhibitor, which activates pyruvate dehydrogenase (PDH), increasing glucose oxidation by promoting an influx of pyruvate into the tricarboxylic acid cycle (2).

DCA affects multiple pathways of intermediary metabolism. It stimulates peripheral glucose utilization and inhibits gluconeogenesis, thereby reducing hyperglycemia in animals and humans with diabetes mellitus. It inhibits lipogenesis and cholesterolgenesis, thereby decreasing circulating lipid and lipoprotein levels in short-term studies of patients with acquired or hereditary disorders of lipoprotein metabolism. By stimulating the activity of pyruvate dehydrogenase, DCA facilitates oxidation of lactate and decreases morbidity in acquired and congenital forms of lactic acidosis (3). It has been shown that DCA reverses the metabolic-electrical remodeling in several cancer lines, increases ROS production, produces hyperpolarized mitochondria, activates NFAT1, induces apoptosis and decreases tumor growth (1). DCA has been found to have antitumor properties in pulmonary epithelial cells (1), breast tumor cells (4), colorectal cancer cells (5) and prostate tumors (6) without affecting normal cells. It has also been shown that 
DCA has an increased effect when combined with other drugs, such as with arsenic trioxide in breast cancer (7), sulindac in lung cancer (8), bortezomib in multiple myeloma (9) or in combination with radiation in prostate cancer (6). Currently, clinical trials are being conducted with DCA to prove its effectiveness (http://clinicaltrial.gov/show/NCT01111097).

All organisms need energy not only to survive but also to prosper and proliferate. Accordingly, properly functioning mitochondria are essential to any cell, including cancer cells (10-12). Metabolic activities of normal cells rely predominately on mitochondrial oxidative phosphorylation (OXPHOS) for energy generation in the form of ATP. On the contrary, cancer cells predominately rely on glycolysis rather than on oxidative phosphorylation (13). There is growing evidence linking cancer with diseases or mutations affecting mitochondrial function and their metabolic pathways (14). Although mitochondrial function and intact mtDNA are essential for cancer cell growth and tumorigenesis, mtDNA mutations and/or reductions in mtDNA copy number that alter the OXPHOS physiology are common features of cancer (15). When mtDNA has a high mutation rate, de novo mtDNA mutations create a mixture of mutant and normal mtDNAs in cells, a state known as heteroplasmy. As the proportion of mutant mtDNAs increases, the energy output capacity of the cell declines until there is insufficient energy to sustain cellular function, termed the bioenergetic threshold. Mitochondria form a reticular network that is constantly undergoing fusion and fission, which is necessary for the maintenance of organelle fidelity (16).

The quality of a mitochondrial population is maintained through mitophagy, a form of specific autophagy in which defective mitochondria are selectively degraded (17). Some antitumor therapies, such as PI3K/mTOR inhibitors, are known to induce autophagy in cancer cells (18). To evaluate autophagy, LC $3 b$ is a commonly used marker because it is an essential component of autophagosomes. The level of the phosphatidylethanolamine-conjugated LC3b (LC3b-II) inserted into the autophagosome membrane and the ratio of LC3b-II/LC3b-I determine the rate of autophagy. However, autophagy itself is a very fast process leading to an immediate fusion of autophagosomes with lysosomes and subsequent degradation of LC3b-II. Blocking the autophagy process in a late phase just prior to lysosome degradation is needed to observe the LC3b-II/LC3b-I ratio. For this reason, $\mathrm{NH}_{4} \mathrm{Cl}$ and chloroquine (CHLQ) have been used to block the completion of autophagy (19).

Due to the importance of mitophagy in mitochondrial quality control and because of the known changes that DCA produces in energy metabolism, we hypothesized that DCA could cause a decrease in mitochondrial density in SH-SY5Y neuroblastoma cells through the activation of mitophagy. As a model, we chose SH-SY5Y cells, originally derived from the SK-N-SH cell line (20), for our study because they are well known and frequently studied. Moreover, neuroblastoma is the most common type of extracranial childhood solid tumor, accounting for $15 \%$ of pediatric cancer-related deaths (21). We used undifferentiated SH-SY5Y cells in this study.

We demonstrate that treatment with DCA $\leq 60 \mathrm{mM}$ stimulates the reorganization of the mitochondrial network in SH-SY5Y cells, leading to shorter and more fragmented mitochondrial filaments. This change in the mitochondrial network was related to an imbalance in the expression of proteins related to mitochondrial dynamics (FIS1), mitochondrial protein degradation (PINK1, Parkin) and autophagy (LC3b). Surprisingly, we did not observe a decrease in mtDNA copy number with DCA treatment. Thus, we provide new insights into the DCA-induced restructuring of the mitochondrial network through mitochondrial dynamics and mitophagy that preserves the nucleoids.

\section{Materials and methods}

Cell culture and experimental design. Neuroblastoma SH-SY5Y cells (ATCC, Manassas, VA, USA) were grown in DMEM (Dulbecco's modified Eagle's medium, Gibco-BRL, Gaithersburg, MD, USA) supplemented with $100 \mathrm{U} / \mathrm{ml}$ penicillin (Sigma-Aldrich, St. Louis, MO, USA), $100 \mu \mathrm{g} / \mathrm{ml}$ streptomycin (Sigma-Aldrich), $5 \mathrm{mM}$ glucose (Sigma-Aldrich), 2 mM L-glutamine (Gibco) and 10\% fetal bovine serum (Gibco) in an atmosphere of $95 \%$ air and $5 \% \mathrm{CO}_{2}$ at $37^{\circ} \mathrm{C}$. The growth medium was replenished every 2 or 3 days. We distributed the $\mathrm{SH}-\mathrm{SY} 5 \mathrm{Y}$ cells into 3 groups: control, $\mathrm{NH}_{4} \mathrm{Cl}$ and chloroquine (CHLQ). $\mathrm{NH}_{4} \mathrm{Cl}$ and CHLQ were added to the experimental design to inhibit mitophagy at different stages, and compared to the control group. The $\mathrm{NH}_{4} \mathrm{Cl}$ group was supplemented with a final concentration of $10 \mathrm{mM} \mathrm{NH}_{4} \mathrm{Cl}$ in the medium. The CHLQ group was supplemented with a final concentration of $50 \mu \mathrm{M}$ CHLQ in the medium. Each group was treated with DCA at 0, 5, 30 and $60 \mathrm{mM}$ for $16 \mathrm{~h}$. Both $\mathrm{NH}_{4} \mathrm{Cl}$ and CHLQ were added at the same time as DCA. All experiments were performed with viable cells after treatment. Therefore, cells in suspension were withdrawn before any analysis.

Number of viable cells. The quantification of viable SH-SY5Y cells was performed as follows: we seeded the cells at a concentration of 150,000 cells/well in three 6 -well plates $(n=3$ for each treatment). After $24 \mathrm{~h}$, we performed a 16-h treatment with DCA. After that, the cells were washed with PBS, trypsinized and quantified with a Countless Automated (Invitrogen, Carlsbad, CA, USA) cell counter using trypan blue.

Mitochondrial copy number. The number of mtDNA copies was assessed via qPCR-RT as described by Alán et al (22). Briefly, total DNA was isolated by phenol-chloroform extraction. We used qPCR and SYBR Green primers recognizing the UCP2 nuclear gene and ND5 mtDNA gene to calculate the corresponding ratio between them, which determines the number of mtDNA copies.

Immunoblotting. All the relative protein quantifications were measured using a protein lysate of SH-SY5Y cells in RIPA buffer (23) supplemented with a protease inhibitor (P8340, Sigma-Aldrich). The total protein concentration of each sample was measured by a BCA (B9643, Sigma-Aldrich) assay (24).

The samples (30 $\mu \mathrm{g}$ of total protein) were separated by SDS-PAGE (12\% polyacrylamide gel) and detected by immunoblotting, as described by Paulson and Laemmli (25). After electrophoresis, the samples were transferred to a PVDF (BioRad, Richmond, CA, USA) membrane for $1 \mathrm{~h}$. Subsequently, blocking was performed using 5\% BSA in TBS-0.05\% Tween-20 on a shaker at room temperature. After blocking, 


\section{Cell viability after DCA treatment}

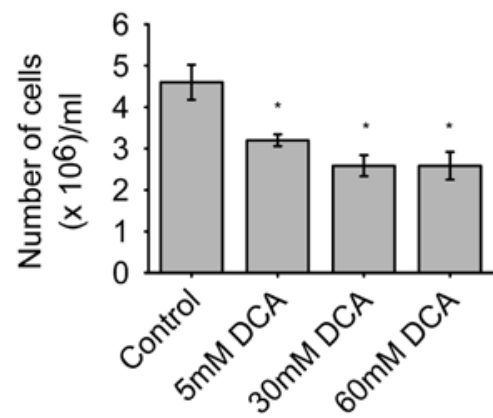

Figure 1. Bar graph represents SH-SY5Y cell growth during the DCA treatment. The cells were initially seeded at a density of 150,000 cells per well. Values are expressed as the mean \pm SEM $(n=3)$. *Significant differences between the control treatment $(\mathrm{P} \leq 0.039)$ by one-way ANOVA (Scheffe).

incubation was performed with LC3b (NB100-2220, Novus Biologicals, Littleton, CO, USA), FIS1 (ALX-210-1037-0100, Enzo Life Science, Farmingdale, NY, USA), OPA1 (612606, BD Biosciences, San José, CA, USA), Tim23 (611222, BD Biosciences), TFAM (ab119684), PARKIN (ab15954) and PINK1 (ab23707) (Abcam, Cambridge, MA, USA) antibodies in blocking buffer with shaking overnight at $4^{\circ} \mathrm{C}$. Actin was used as the loading control. The actin primary antibody (ab3280, Abcam) was diluted 1:1,000 in blocking buffer and also incubated as above. In all cases, appropriate secondary peroxidase-conjugated antibodies, anti-mouse or anti-rabbit, were applied at a concentration of 1:5,000 in TBS-T for $1 \mathrm{~h}$ with shaking at room temperature. Image J software was used to perform densitometry and relative quantification (26).

Confocal microscopy. SH-SY5Y cells were cultured for 2 days on glass cover slips coated with poly-L-lysine. DCA was administered in the culture medium for $16 \mathrm{~h}$. The mitochondria specific fluorescent dye tetramethylrhodamine ethyl ester (TMRE) was used to investigate mitochondrial structure. TMRE is a lipophilic, cell permeable, cationic, non-toxic, fluorescent dye that specifically stains live mitochondria. TMRE accumulated specifically in the mitochondria in proportion to the mitochondria membrane potential (27). We incubated TMRE at a final concentration of $50 \mu \mathrm{M}$ in the cell culture medium for $1 \mathrm{~min}$ and then exchanged the medium for fresh culture medium. Cells were then placed on a microscope slide, which was imaged with an inverted confocal fluorescent Leica TCS SP2 AOBS microscope with a PL APO 100x/1.40-0.70 oil immersion objective (a pinhole of $1 \mathrm{AU}$ ). TMRE was excited at $543 \mathrm{~nm}$ with a $1.2-\mathrm{mW}$ HeNe laser. All images of living cells were taken in $95 \%$ air and $5 \% \mathrm{CO}_{2}$ at $37^{\circ} \mathrm{C}$ in a microscope incubation chamber.

Statistical analysis. The results are reported as the mean \pm SEM. The means were compared with one-way or two-way ANOVA $(\mathrm{P} \leq 0.05)$ using SPSS for Windows v. 19.0 software (SPSS Inc., Chicago, IL, USA).

\section{Results}

DCA decreases the number of viable cells. The SH-SY5Y cell viability is illustrated in Fig. 1. The control group had a

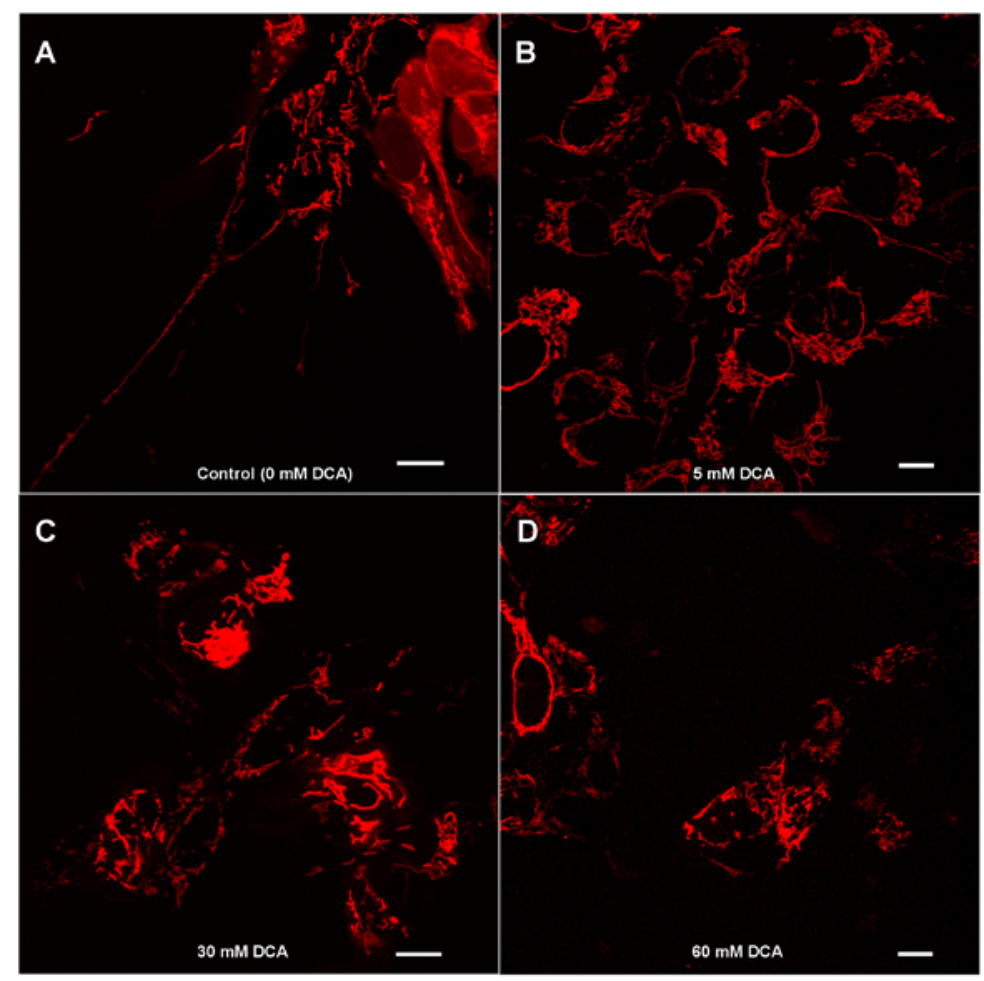

Figure 2. Microscopy images of SH-SY5Y neuroblastoma cells treated with different DCA doses. (A) Control (0 mM), (B) $5 \mathrm{mM}$, (C) $30 \mathrm{mM}$ and (D) $60 \mathrm{mM}$ DCA treatment. Scale bars represent $10 \mu \mathrm{m}$. The control group images have a mitochondrial network with interconnected and long filaments. With increasing DCA concentrations, a higher presence of shorter and more fragmented filaments in the mitochondrial network was observed. No significant change in cell size was observed in the DCA treatment groups when compared with controls. 
maximum of $4.6 \times 10^{5}$ viable cells/well, while the $5 \mathrm{mM}$ DCA dose had $3.2 \times 10^{5}$ cells/well, and two doses of 30 and $60 \mathrm{mM}$ DCA both had an average of $2.6 \times 10^{5}$ cells/well. Treatment with the lowest concentration of DCA $(5 \mathrm{mM})$ significantly decreased the number of viable cells $(\mathrm{P}<0.05)$, as did the other two higher doses of DCA, where the differences were even higher than in the $5 \mathrm{mM}$ DCA group.

The concentration of 30 and $60 \mathrm{mM}$ DCA had a similar effect on the number of viable cells. It is noteworthy that these differences in the number of viable cells were easily observable under a microscope. We observed cells in suspension after DCA treatment, which is indicative of cell death or apoptosis. This phenomenon was observed in all series of wells that were treated with DCA.

DCA induces morphology changes in the mitochondrial network. Confocal microscopy showed that no significant change in the cell size was observed in the DCA-treated groups compared with controls. The control group tended to have a mitochondrial structure that was long and contained interconnected filaments. As the DCA concentration increased, the mitochondrial network contained shorter and more fragmented filaments. At higher DCA concentrations, there were clustered mitochondria filaments more frequently present, causing the filaments of the mitochondrial network not to be distributed homogeneously within the cell cytosol (Fig. 2).

DCA at high doses increases the LC3b ratio. LC3b-I and LC3b-II were detected in SH-SY5Y cells. In the control group, we found that the concentration of LC3b-I increases with increasing DCA concentrations. As expected, we did not detect LC3b-II in this group at any DCA concentration. In the $\mathrm{NH}_{4} \mathrm{Cl}$ treatment, the LC3b-I levels were similar and LC3b-II levels were decreased, as compared to the control sample. The CHLQ group exhibited a decrease in LC3b-I levels and an increase in LC3b-II levels with increasing DCA concentrations (Fig. 3A and B).

In addition, we calculated the LC3b-II/LC3b-I ratio, which indicates how much LC3B-I was transformed to LC3B-II during autophagy and gives an approximation of the level of autophagy induced by the treatment. The LC3B-II/LC3B-I ratio was very small and no significant changes were found in the control group. However, in the $\mathrm{NH}_{4} \mathrm{Cl}$ or CHLQ treatments, increasing ratios were obtained with increasing DCA concentrations, especially using CHLQ as autophagy blocker (Fig. 3C).

DCA produces an imbalance of OPA1 isoforms and reduces FISI and Tim 23 protein levels. We detected a redistribution of both OPA1 isoforms. The two bands of OPA1, corresponding to OPA $1 \mathrm{c}, \mathrm{d}$ and e isoforms ( $92 \mathrm{kDa}$ ) and OPA1 a and b isoforms $(100 \mathrm{kDa})$, were examined (27). Treatment with $5 \mathrm{mM}$ DCA did not have an effect on the OPA1 bands. However, treatment with $30 \mathrm{mM}$ DCA resulted in an increase in both OPA1 bands, while treatment with $60 \mathrm{mM}$ DCA increased only the $92-\mathrm{kDa}$ OPA1 isoform, compared to the control group (Fig. 4A and B).

$\mathrm{NH}_{4} \mathrm{Cl}$ treatment produced a slight increase in both bands of OPA1. The co-treatment of $\mathrm{NH}_{4} \mathrm{Cl}$ with $\leq 30 \mathrm{mM}$ DCA caused a slight increase in the $92-\mathrm{kDa}$ OPA1 isoform. In

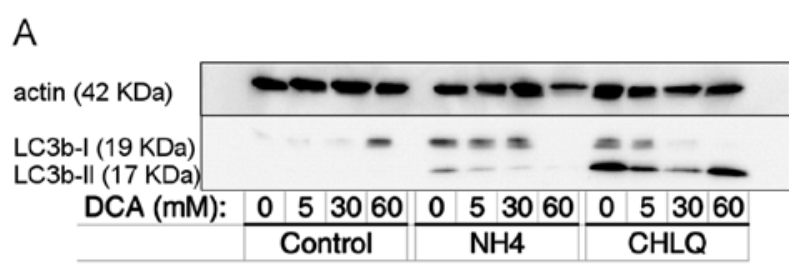

\section{B Relative protein expression of LC3b-I and LC3b-II after DCA treatment}
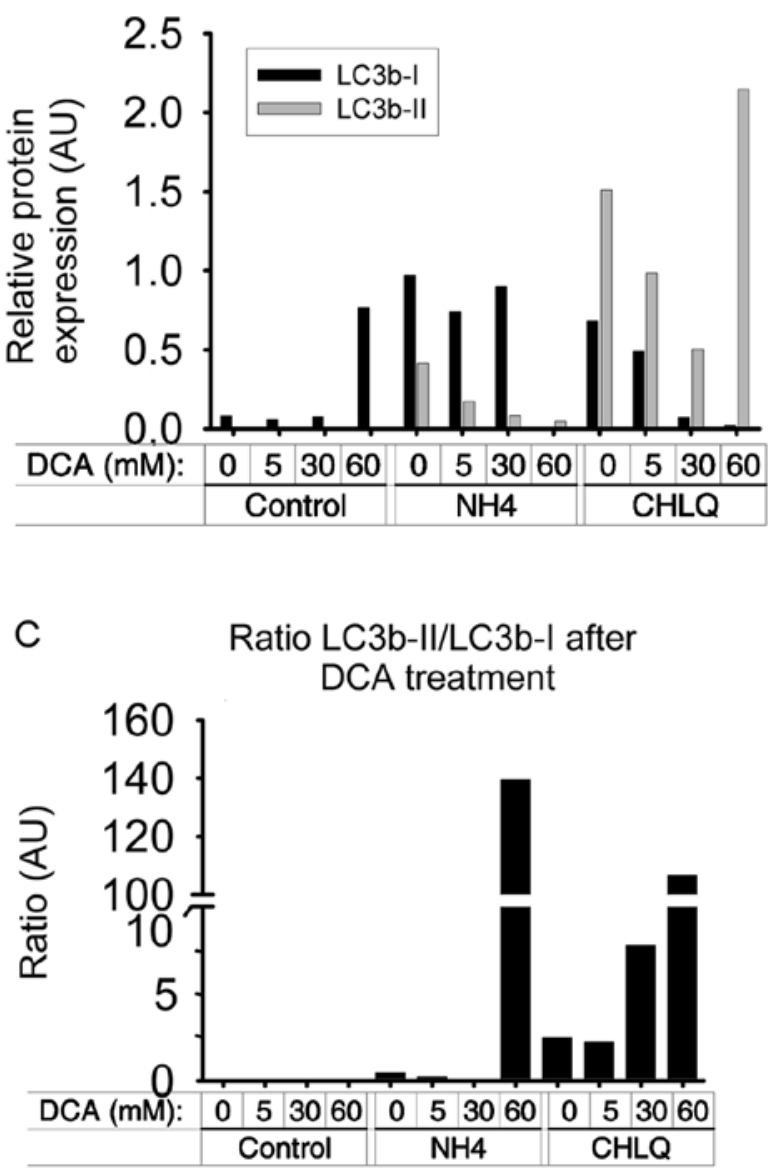

Figure 3. (A) Western blot images of actin and LC3b (I and II isoforms) proteins. There are three groups of treatments: the control group that received only DCA as treatment, the group represented by NH4, which was co-treated with a final concentration of $10 \mathrm{mM} \mathrm{NH}_{4} \mathrm{Cl}$ and DCA, and the group represented by CHLQ, which was co-treated with a final concentration of $50 \mu \mathrm{M}$ CHLQ and DCA. Note that with the dose of $60 \mathrm{mM} \mathrm{DCA}+\mathrm{NH}_{4} \mathrm{Cl}$, we observed a high proportion of apoptotic cells, which is reflected in the levels of actin protein. (B) Quantification of the bands was performed by densitometry using ImageJ software. Relative LC $3 b$ protein expression calculations represented in the graph were performed using actin as a reference protein. (C) The LC3b-II (17 kDa)/LC3b-I (19 kDa) ratios were used to analyze the autophagy levels induced by the treatment.

contrast, co-treatment of $\mathrm{NH}_{4} \mathrm{Cl}$ with $60 \mathrm{mM}$ DCA resulted in a significant decrease in the two OPA1 bands, especially in the higher one. In fact, this combination proved to be toxic to the cells by causing apoptosis, as indicated by the actin band (Fig. 4A and B).

CHLQ treatment caused a reduction in the two OPA1 bands, which was more pronounced for the higher band. The co-treatment of CHLQ with $5 \mathrm{mM}$ DCA did not affect OPA1 


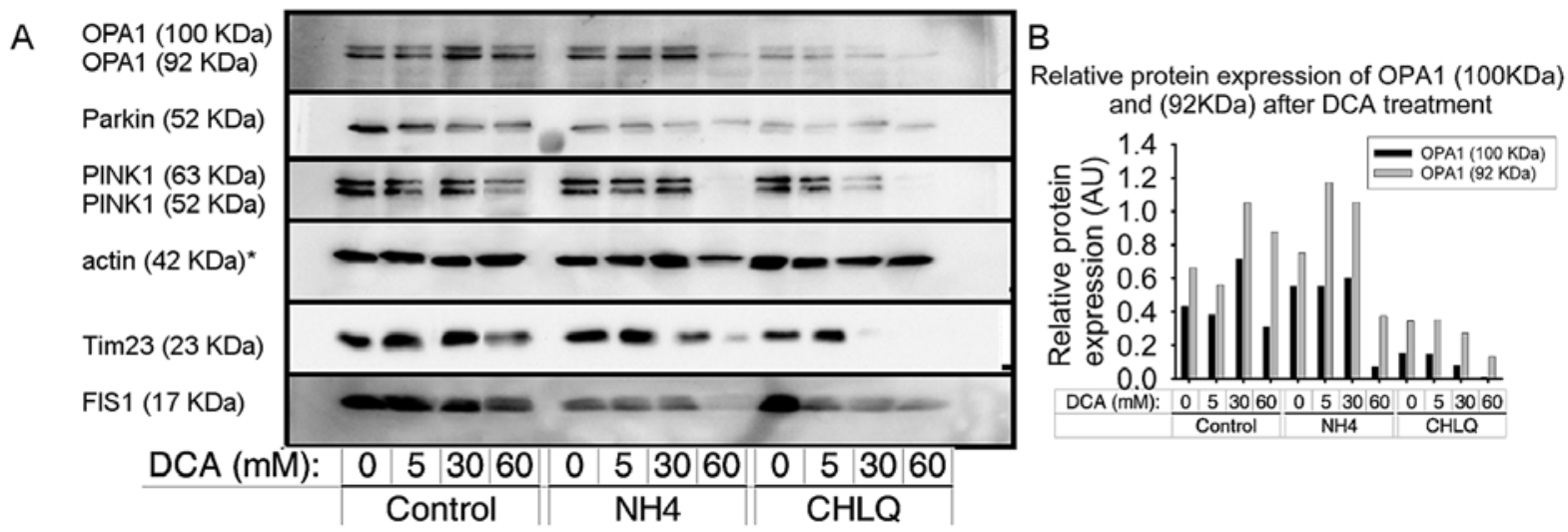

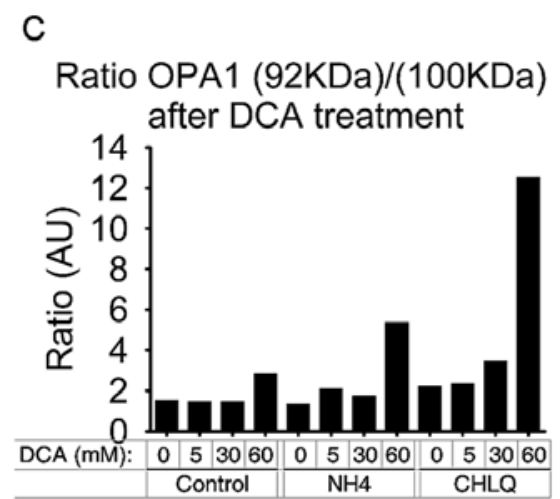

$\mathrm{F}$

Ratio PINK1 (52KDa)/PINK1 (63KDa)

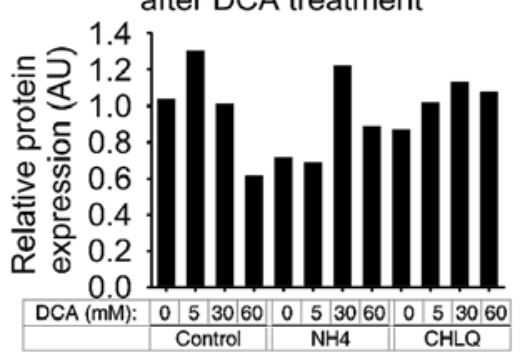

D

\section{Relative protein expression of} Parkin after DCA treatment

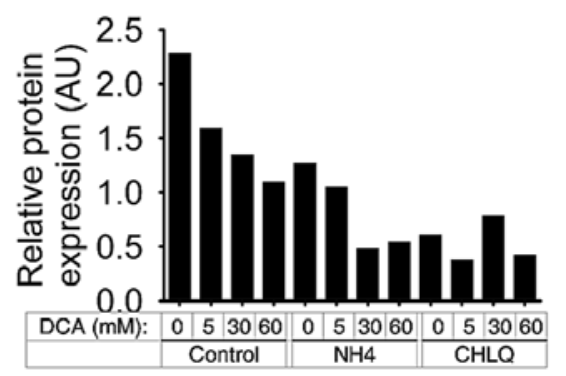

G

Relative protein expression of Tim23 after DCA treatment

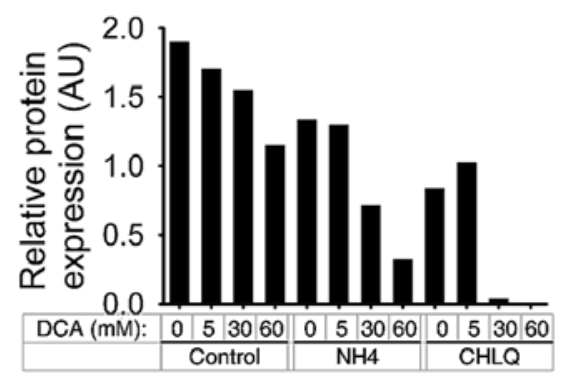

E

Relative protein expression of PINK (63KDa) and (52KDa) after

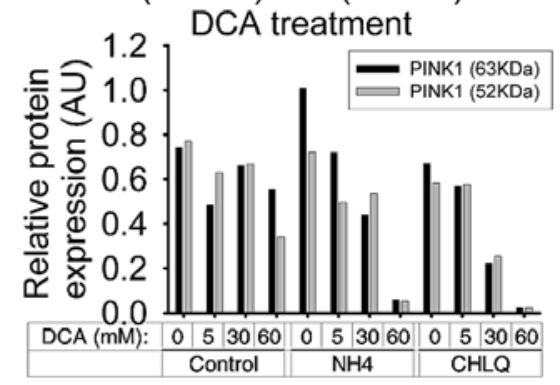

$\mathrm{H}$

Relative protein expression of FIS1 after DCA treatment

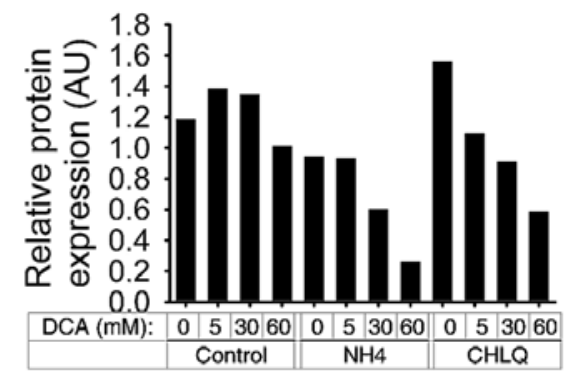

Figure 4. (A) Western blot images of proteins involved in mitochondrial dynamics, Tim 23 and actin proteins sorted by molecular weight. There are three groups of treatments: the control group that received only DCA as treatment, the group represented by NH4, which was co-treated with a final concentration of $10 \mathrm{mM} \mathrm{NH}_{4} \mathrm{Cl}$ and DCA, and the group represented by CHLQ, which was co-treated with a final concentration of $50 \mu \mathrm{M} \mathrm{CHLQ}$ and DCA. Note that at the dose of $60 \mathrm{mM} \mathrm{DCA}+\mathrm{NH}_{4} \mathrm{Cl}$, we observed a high proportion of apoptotic cells, which is reflected in the levels of actin protein. "All calculations presented in the graphs of the relative protein expression were performed using actin as a reference protein. Quantification of the bands was performed by densitometry using ImageJ software. (B) Graphical representation of the relative expression of the two isoforms of OPA1 (100 kDa) and (92 kDa). (C) The OPA1 (92 kDa)/ OPA1 (100 kDa) ratios are presented. (D) Graphical representation of the relative expression of the Parkin protein (52 kDa). (E) The relative expression of the PINK1 protein (63- and 52-kDa isoforms) are represented. (F) The PINK1 (52 kDa)/PINK1 (63 kDa) ratios are presented. (G) Graphical representation of the relative expression of the Tim $23(23 \mathrm{kDa})$. (H) The relative expression of the FIS1 protein $(17 \mathrm{kDa})$ is presented.

expression; however, at higher DCA concentrations (30 and $60 \mathrm{mM})$, there was a slight decrease in both OPA1 bands. At $60 \mathrm{mM}$ DCA, the 100-kDa isoform was non-existent (Fig. 4B).

We calculated the OPA1 isoform ratio [OPA1 $(92 \mathrm{kDa}) /$ OPA1 $(100 \mathrm{kDa})]$ and found that it increased after the $60-\mathrm{mM}$ DCA treatment. Likewise, the co-treatment with $\mathrm{NH}_{4} \mathrm{Cl}$ or CHLQ amplified this effect (Fig. 4C).

FIS1 protein levels were decreased by DCA treatment in SH-SY5Y cells in all groups. FIS1 protein levels tended to slightly increase with 5 and $30 \mathrm{mM}$ DCA treatment in the control group, despite FIS1 protein levels decreasing slightly at higher DCA concentrations $(60 \mathrm{mM})$ in the control group. In the two groups that were co-treated with mitophagy blocking agents, FIS1 protein levels exhibited a similar profile. FIS1 protein levels decreased with increasing DCA concentrations in a dose-dependent manner. The $\mathrm{NH}_{4} \mathrm{Cl}$ treatment showed slightly lower FIS1 protein levels, down to $24 \%$ (Fig. 4A and $\mathrm{H}$ ). 


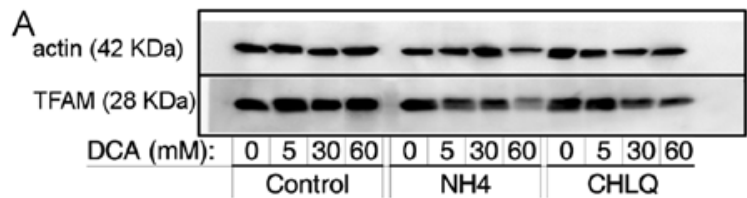

B Relative protein expression of TFAM after DCA treatment
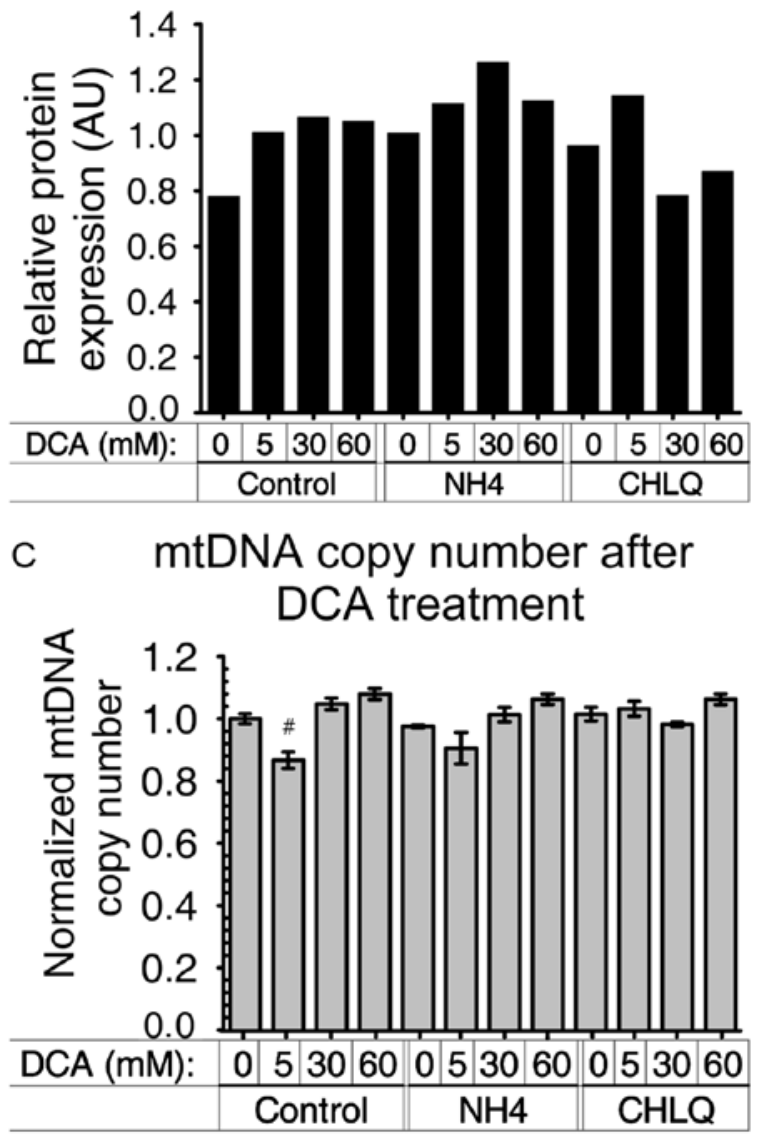

Figure 5. (A) Western blot images of actin and TFAM proteins. There are three groups of treatments: the control group that received only DCA as treatment, the group represented by $\mathrm{NH} 4$, which was co-treated with a final concentration of $10 \mathrm{mM} \mathrm{NH}_{4} \mathrm{Cl}$ and DCA, and the group represented by CHLQ, which was co-treated with a final concentration of $50 \mu \mathrm{M}$ CHLQ and DCA. Note that at the dose of $60 \mathrm{mM} \mathrm{DCA}+\mathrm{NH}_{4} \mathrm{Cl}$, we observed a high proportion of apoptotic cells, which is reflected in the levels of actin protein. (B) Quantification of the bands was performed by densitometry using Image J software. Relative TFAM protein level calculations presented in the graph were performed using actin as a reference protein. (C) Normalized mitochondrial copy numbers are presented for each treatment including co-treatment with the autophagy blockers $\mathrm{NH}_{4} \mathrm{Cl}$ and CHLQ. The values are expressed as the mean \pm SEM $(n=3)$. "Significant differences compared to the control treatment, as determined by two-way ANOVA $(\mathrm{P}=0.07)$.

Tim23 decreased with increasing DCA concentrations in a dose-dependent manner in all the groups. This effect was moderate in the control group and more pronounced in the $\mathrm{NH}_{4} \mathrm{Cl}$ and CHLQ groups. Thus, the co-treatment of DCA with autophagy blockers led to a further decrease in Tim23 levels, especially with CHLQ treatment (Fig. 4A and G).

DCA decreases the PINK1 and Parkin protein levels. The results obtained by immunoblotting showed that DCA treat- ment caused a decrease in the protein levels of PINK1 and Parkin in SH-SY5Y cells. These decreases were observed in the control group and both the $\mathrm{NH}_{4} \mathrm{Cl}$ and CHLQ groups, in which the mitophagy event is blocked by different mechanisms at a late phase (Fig. 4A).

The protein levels of the two PINK1 isoforms were decreased at the highest dose of DCA $(60 \mathrm{mM})$. When DCA treatment was applied in combination with mitophagy blockers $\left(\mathrm{NH}_{4} \mathrm{Cl}\right.$ and $\left.\mathrm{CHLQ}\right)$, we observed a decrease in the two PINK1 isoforms in a dose-dependent manner (Fig. 4E). In these two groups, the expression of PINK1 with the $60 \mathrm{mM}$ DCA treatment was almost non-existent. In the combined treatment of $60 \mathrm{mM}$ DCA and $\mathrm{NH}_{4} \mathrm{Cl}$, there was a high percentage of cells undergoing apoptosis, which can be seen in the lower density of the actin band (Fig. 4A). We calculated the ratios between the two PINK1 isoforms, PINK1 (53 kDa)/PINK1 (63 kDa), to determine if they correlated to drug concentration. The PINK1 isoform ratios showed two trends. The first trend was found in the control group, where an increasing DCA concentration reduced the ratios between the two PINK1 isoforms. The second trend was that the $\mathrm{NH}_{4} \mathrm{Cl}$ and CHLQ treatment groups exhibited a slight increase in the PINK1 (53 kDa)/PINK1 (63 kDa) ratio with increasing DCA concentrations (Fig. 4F).

The Parkin protein levels decreased with increasing DCA concentrations in a dose-dependent manner for the control group. Treatment with autophagy-blocking agents $\left(\mathrm{NH}_{4} \mathrm{Cl}\right.$ or CHLQ) without DCA produced a significant decrease in Parkin protein levels, decreasing by half and two-thirds, respectively. The co-treatment of $\mathrm{NH}_{4} \mathrm{Cl}$ or CHLQ with DCA caused downregulation of the Parkin protein compared to controls for their respective treatment groups (Fig. 4D).

DCA does not alter mitochondrial DNA density in SH-SY5Y cells. We used TFAM as a marker of mtDNA density. The results obtained by immunoblotting did not show a significant difference in TFAM protein levels due to DCA treatment or the co-treatment with $\mathrm{NH}_{4} \mathrm{Cl}$ or CHLQ (Fig. 5A and B).

To verify these results, we also analyzed the number of mitochondrial DNA copies by real-time PCR. Fig. 5C shows the mitochondrial DNA copy number. No significant differences were found. Only a small difference was obtained between the control group treated with $5 \mathrm{mM}$ DCA and the untreated control, with a P-value of 0.07 using two-way ANOVA (Fig. 5C).

\section{Discussion}

We treated neuroblastoma SH-S5YS cells with DCA. The concentration of $60 \mathrm{mM}$ was chosen as the maximum dose because previous studies reported that DCA is relatively inactive in different cells lines when used at low doses $(5,28)$, despite the fact that such a concentration is unlikely to be achieved in vivo. To perform an accurate analysis, we also studied two additional groups that were co-treated with DCA and the mitophagy-blocking compounds $\mathrm{NH}_{4} \mathrm{Cl}$ or CHLQ. The purpose was to observe the accumulation of mitophagy marker proteins and analyze the changes in these proteins after the DCA treatment. $\mathrm{NH}_{4} \mathrm{Cl}$ inhibits lysosomal proteolysis and is independent of the type autophagy that delivers substrates to lysosomes (29). CHLQ is a well-known inhibitor of the final 
step in the autophagy pathway (30). However, both $\mathrm{NH}_{4} \mathrm{Cl}$ and CHLQ may have other side effects. For example, CHLQ has been shown to reduce tumor growth, hypoxia, cancer cell invasion, and metastasis but facilitate chemotherapy delivery and the tumor milieu by improving tumor perfusion and oxygenation (31).

Dose-dependent decreases in membrane proteins such as Tim23, PINK1, Parkin and FIS1 suggest that autophagy is involved in the degradation of mitochondrial membrane components, a process known as mitophagy. This is most visible for Parkin and Tim 23 in the control groups without autophagy blockers. In our experiments, the two autophagy blockers did not have identical effects, although both had similar effects.

DCA has high bioavailability, as it can easily pass through the plasma and mitochondrial membranes via the monocarboxylate and pyruvate transporter systems, respectively. It also easily crosses the blood-brain barrier and may concentrate in the mitochondria $(32,33)$.

Recently, it has been demonstrated that DCA induces autophagosome formation and autophagy due to ROS accumulation in LoVo cells. Although, four additional colorectal cancer cell lines, which are similar to LoVo cells, proved to be less sensitive to DCA treatment (34). Moreover, it has been reported that inhibitors of autophagy were able to promote apoptosis induced by anticancer drugs, which suggested that autophagy could have a protective role in cancer cells $(34,35)$.

In this report, we studied how sensitive DCA treatment is in neuroblastoma SH-SY5Y cells. Initially, we assessed the sensitivity of SH-SY5Y cells to DCA by a cell viability experiment. As expected, we found that DCA led to a decrease in viable cells. Surprisingly, this decrease was equal to the decrease seen with two higher DCA doses. Thus, the $60 \mathrm{mM}$ dose of DCA produced the same effects as the $30 \mathrm{mM}$ DCA dose. It is noteworthy that even the lowest DCA dose $(5 \mathrm{mM})$ caused a significant decrease in the number of viable cells. The difference in the number of viable cells compared to the control group is represented by the cells that have died or are undergoing apoptosis. Cells were frequently detached and found in suspension, indicating that a percentage of cells were undergoing apoptosis. This was a feature that we observed only in cells treated with DCA, not in control cells. Nevertheless, all experiments were performed with the viable cells after treatment. Thus, the cells in suspension were not used in any analysis.

Our results confirmed previous reports on the pro-apoptotic and anti-proliferative effects of DCA in human non-small cell lung cancer (A549), glioblastoma (MO59K), breast cancer (MFC-7) (1) and endometrial cancer cells (36). Moreover, the use of autophagy inhibitors such as 3-methyladenine (3-MA) or, in our case, $\mathrm{NH}_{4} \mathrm{Cl}$ or CHLQ in combination with DCA has been reported to significantly enhance DCA-induced apoptosis (34). Contrary to our observations, a recent study of DCA effects in colorectal and prostate cancer cell lines reported a minimal effect of DCA on apoptosis. Even so, the authors noted that despite not observing apoptosis, they observed an increase in LC3b-II expression (2). This dual behavior of DCA, stimulating apoptosis in some cases and not in others, could be cell type-dependent and due to differences in the expression of PDK isozymes of the examined cancer cells (37).
Our data suggest that a certain percentage of apoptosis is induced by DCA treatment, but the percentage of apoptosis is not increased in a dose-dependent response as we initially thought. A possible explanation to why some cells undergo apoptosis and others do not when treated with the same concentration of DCA could be the conclusion arrived to by Vella et al (37), reporting that the effects of DCAs are restricted to undifferentiated, malignant, fully cycling neuroblastoma cells, whereas DCA does not affect the proliferation rate of more differentiated, poorly malignant neuroblastoma cells (37).

We further related the effects of DCA treatment to mitophagy by evaluating a set of proteins involved in mitochondrial network dynamics and protein degradation. We analyzed the copy number of mitochondrial DNA and the mitochondrial network morphology.

It has been reported that DCA treatment reduced the mitochondrial membrane potential in a dose-dependent manner (1). We found that there was a slight decrease in the intensity emitted by TMRE in cells that were treated with the highest dose of DCA. In all cases, it was sufficient to evaluate the mitochondrial network. One of our findings was that increasing DCA doses led to the mitochondrial network becoming more condensed. The long filaments of the mitochondrial network were a feature unique to the control group (Fig. 2).

The LC3b proteins are involved in phagophore formation and are commonly used markers for examining autophagy. A high ratio of LC3b-I to LC3b-II is a typical finding in cells of neuronal origin (38). For instance, SH-SY5Y neuroblastoma cell lines display only a slight increase in LC3b-II after nutrient deprivation, whereas LC3b-I is clearly reduced. This is likely related to a high basal autophagic flux, as suggested by the higher increase in LC3b-II when cells are treated with $\mathrm{NH}_{4} \mathrm{Cl}(39,40)$, although cell-specific differences in transcriptional regulation of LC3b may also play a role. The pattern of LC3b-I to LC3b-II conversion seems to be not only cellspecific but also related to certain cellular stresses. SH-SY5Y cells displayed a strong increase in LC3b-II when treated with CHLQ. The ratio LC3b-II/LC3b-I was very low and unchanged in the control group because the high turnover of LC3b in these cells. This ratio was unchanged at low doses but increased at $30 \mathrm{mM}$ DCA and increased drastically at $60 \mathrm{mM}$ DCA during the co-treatment with mitophagy blockers (Fig. 3). These results suggest that a high DCA concentration stimulates phagophore formation, leading to autophagy after $16 \mathrm{~h}$ of treatment in SH-SY5Y neuroblastoma cells. Therefore, we suggest that the loss of cell viability due to the DCA treatment at low concentrations observed after $16 \mathrm{~h}$ is not caused by mitophagy in SH-SY5Y cells.

Recent in vitro studies suggested that PINK1 and Parkin cooperate in a pathway to regulate mitochondrial quality via degradation of dysfunctional mitochondria by mitophagy (41). For mitochondrial degradation, PINK1 reportedly recruits Parkin from the cytoplasm to damaged mitochondria in a manner that is dependent on the mitochondria membrane potential (42). Moreover, PINK1 and Parkin initiate the removal of mitochondrial proteins via the ubiquitinproteasome system (43). It has been reported that deficits in PINK1 and Parkin alter the balance between mitochondrial fission and fusion (43). Our results show that a higher DCA concentration reduced the presence of both PINK1 isoforms 
(Fig. 4A, E and F). Moreover, Parkin was decreased in a doseresponse manner with increasing DCA concentrations (Fig. 4A and D). This result confirmed previous studies in which knockdown of PINK1 in HeLa cells and human neuronal SH-SY5Y cells caused mitochondrial fragmentation, an effect that was reversed by overexpression of Parkin $(44,45)$. Thus, our data indicate that lower protein expression of PINK1 and Parkin due to DCA treatment induced further fragmentation of the mitochondrial network in SH-SY5Y cells, as observed in the microscopy images (Fig. 2).

Mitochondrial fission is essential for autophagy. We have shown that the FIS1 levels decreased in a dose-dependent manner after the DCA treatment. This decrease in FIS1 was most evident in the groups in which the process of autophagy was blocked with $\mathrm{NH}_{4} \mathrm{Cl}$ or CHLQ (Fig. 4A and $\mathrm{H}$ ). It has been previously reported that silencing FIS1 with RNAi is associated with an inhibition of mitochondrial autophagy and the accumulation of damaged mitochondrial material, leading to a decrease in metabolic function and insulin secretion. Furthermore, this same study showed that silencing FIS1 had mild changes in mitochondrial structure (46). Contrary to our initial hypothesis, DCA decreases FIS1 and, therefore, mitochondrial fission in SH-SY5Y cells and presumably protects against mitophagy.

When comparing $(0 \mathrm{mM})$ DCA controls among all three groups (control, $\mathrm{NH}_{4} \mathrm{Cl}, \mathrm{CHLQ}$ ), blocking terminal phases of autophagy itself decreases the relative protein expression of Parkin and Tim23. However, a much more significant shift was observed when comparing the control, $\mathrm{NH}_{4} \mathrm{Cl}$ and $\mathrm{CHLQ}$ group cells treated by the most toxic dose of DCA $(60 \mathrm{mM})$. Thus, blocking autophagy leads to a much stronger decrease in PINK1, Parkin, FIS1, OPA1 and Tim23.

OPA1 is a dynamin-related GTPase protein involved in inner mitochondrial membrane fusion, which maintains the connected mitochondrial network and correct cristae morphology (47). OPA1 expression increases during mitochondrial elongation $(48,49)$. Though OPA1 protein levels decreased with higher DCA concentrations, especially in combination with mitophagy blockers, the levels of both OPA1 isoforms remained unchanged with other DCA treatments, leading us to conclude that DCA produces a minimal effect on OPA1 protein expression. Only the higher DCA dose induced mitochondrial fragmentation, which is partly due to the decrease in the smaller OPA1 isoform. This is similar to a report in which OPA1-depleted neuronal cells exhibited impaired mitochondrial hyperfilamentation and synaptic number (50).

The short and long isoforms of OPA 1 arise from proteolytic cleavage, and their ratio may serve as a stress indicator (51). The OPA1 ratio of short to long isoforms was increased with a higher concentration of DCA. Interestingly, OPA1 short/long isoform ratios were unaltered from 0 to $30 \mathrm{mM}$ DCA. This result indicated that the higher dose of DCA was a unique dose that produced stress in the cells.

We found that neither the mitochondrial DNA copy number (Fig. 5C) nor TFAM protein levels (Fig. 5A and B) were modified by the DCA treatment. TFAM protein levels are generally used as a mitochondrial density marker because TFAM is part of mitochondrial DNA-containing structures called nucleoids (52). Thus, we confirmed a previous study reporting that the treatment of $C$. elegans with DCA did not affect the mitochondrial DNA copy number as examined by real-time PCR (53). This is in contrast to previous reports that DCA treatment stimulates the removal of mitochondria, resulting in the mitophagy in cancer cells, such as LoVo cells (34) or colorectal cells (2). We have two possible explanations for these results. One is that the effects produced by DCA did not affect mitochondrial density and thus did not induce mitophagy, but DCA does affect the removal of other organelles of the cell in the form of autophagy. The other explanation is that DCA reduced the number of mitochondria maintaining the mtDNA density. In other words, DCA induced partial mitophagy in which the nucleoids are preserved.

We also analyzed Tim 23 protein levels. Tim 23 is a component of the TIM23 complex, which is present in the inner mitochondrial membrane. Because of its localization, we used Tim23 as a marker of the mitochondrial inner membrane (54). Tim 23 protein levels decreased with increasing DCA doses. Co-treatment with autophagy blockers increased this effect. These results indicate that DCA decreased mitochondria numbers leading to mitophagy, which was also indicated by the $\mathrm{LC} 3 \mathrm{~b}$ ratio.

We suggest that DCA induces partial mitophagy preserving nucleoids. It is for this reason we could not detect a decrease in the number of mtDNA copies nor TFAM protein levels.

In conclusion, in this study, we show that DCA causes cell death in SH-SY5Y neuroblastoma cells. The surviving cells after DCA treatment exhibit an altered mitochondrial network morphology that was made up of shorter and more fragmented filaments. We related this mitochondrial network restructuring to changes in FIS1, PINK1, Parkin and LC3b protein levels. Although DCA treatment did not change the number of mtDNA copies and TFAM levels, we suggest that DCA causes partial mitophagy preserving the nucleoids.

Additionally, our data show that autophagy in general is a key mechanism to maintain proper mitochondrial content by balancing fission and fusion processes. Fission and fusion are regulated by mitophagy proteins (PINK1 and Parkin) and FIS1, and the mitochondrial density can be monitored by internal membrane components such as Tim23. Disturbances caused by blocking terminal phases of autophagy with $\mathrm{NH}_{4} \mathrm{Cl}$ and CHLQ decreases the levels of these proteins. These protein levels are further decreased when co-treated with $\mathrm{NH}_{4} \mathrm{Cl}$ or CHLQ and DCA in comparison to negative controls, suggesting that autophagy plays a protective role against the toxic effects of DCA.

\section{Acknowledgements}

This study was supported within the project The Centre of Biomedical Research (CZ.1.07/2.3.00/30.0025). This study was also co-funded by the European Social Fund and the state budget of the Czech Republic.

\section{References}

1. Bonnet S, Archer SL, Allalunis-Turner J, Haromy A, Beaulieu C, Thompson R, Lee CT, Lopaschuk GD, Puttagunta L, Bonnet S, et al: A mitochondria- $\mathrm{K}^{+}$channel axis is suppressed in cancer and its normalization promotes apoptosis and inhibits cancer growth. Cancer Cell 11: 37-51, 2007. 
2. Lin G, Hill DK, Andrejeva G, Boult JK, Troy H, Fong AC, Orton MR, Panek R, Parkes HG, Jafar M, et al: Dichloroacetate induces autophagy in colorectal cancer cells and tumours. Br J Cancer 111: 375-385, 2014

3. Stacpoole PW: The pharmacology of dichloroacetate. Metabolism 38: 1124-1144, 1989.

4. Sun RC, Fadia M, Dahlstrom JE, Parish CR, Board PG and Blackburn AC: Reversal of the glycolytic phenotype by dichloroacetate inhibits metastatic breast cancer cell growth in vitro and in vivo. Breast Cancer Res Treat 120: 253-260, 2010.

5. Madhok BM, Yeluri S, Perry SL, Hughes TA and Jayne DG: Dichloroacetate induces apoptosis and cell-cycle arrest in colorectal cancer cells. Br J Cancer 102: 1746-1752, 2010.

6. Cao W, Yacoub S, Shiverick KT, Namiki K, Sakai Y, Porvasnik S, Urbanek C and Rosser CJ: Dichloroacetate (DCA) sensitizes both wild-type and over expressing Bcl-2 prostate cancer cells in vitro to radiation. Prostate 68: 1223-1231, 2008.

7. Sun RC, Board PG and Blackburn AC: Targeting metabolism with arsenic trioxide and dichloroacetate in breast cancer cells. Mol Cancer 10: 142, 2011.

8. Ayyanathan K, Kesaraju S, Dawson-Scully K and Weissbach H: Combination of sulindac and dichloroacetate kills cancer cells via oxidative damage. PLoS One 7: e39949, 2012.

9. Sanchez WY, McGee SL, Connor T, Mottram B, Wilkinson A, Whitehead JP, Vuckovic S and Catley L: Dichloroacetate inhibits aerobic glycolysis in multiple myeloma cells and increases sensitivity to bortezomib. Br J Cancer 108: 1624-1633, 2013.

10. Fantin VR, St-Pierre J and Leder P: Attenuation of LDH-A expression uncovers a link between glycolysis, mitochondrial physiology, and tumor maintenance. Cancer Cell 9: 425-434, 2006.

11. Gaude E and Frezza C: Defects in mitochondrial metabolism and cancer. Cancer Metab 2: 10, 2014.

12. Weinberg F, Hamanaka R, Wheaton WW, Weinberg S, Joseph J, Lopez M, Kalyanaraman B, Mutlu GM, Budinger GR and Chandel NS: Mitochondrial metabolism and ROS generation are essential for Kras-mediated tumorigenicity. Proc Natl Acad Sci USA 107: 8788-8793, 2010.

13. Singleterry J, Sreedhar A and Zhao Y: Components of cancer metabolism and therapeutic interventions. Mitochondrion 17 50-55, 2014.

14. Astuti D, Hart-Holden N, Latif F, Lalloo F, Black GC, Lim C, Moran A, Grossman AB, Hodgson SV, Freemont A, et al: Genetic analysis of mitochondrial complex II subunits SDHD, SDHB and SDHC in paraganglioma and phaeochromocytoma susceptibility. Clin Endocrinol (Oxf) 59: 728-733, 2003.

15. Wallace DC: Mitochondria and cancer. Nat Rev Cancer 12: 685-698, 2012

16. Youle RJ and Karbowski M: Mitochondrial fission in apoptosis Nat Rev Mol Cell Biol 6: 657-663, 2005.

17. Chen $\mathrm{H}$ and Chan DC: Mitochondrial dynamics - fusion, fission, movement, and mitophagy - in neurodegenerative diseases. Hum Mol Genet 18 (R2): R169-R176, 2009.

18. Raynaud FI, Eccles S, Clarke PA, Hayes A, Nutley B, Alix S, Henley A, Di-Stefano F, Ahmad Z, Guillard S, et al: Pharmacologic characterization of a potent inhibitor of class I phosphatidylinositide 3-kinases. Cancer Res 67: 5840-5850, 2007.

19. Klionsky DJ, Abdalla FC, Abeliovich H, Abraham RT, AcevedoArozena A, Adeli K, Agholme L, Agnello M, Agostinis P, Aguirre-Ghiso JA, et al: Guidelines for the use and interpretation of assays for monitoring autophagy. Autophagy 8: 445-544, 2012

20. Biedler JL, Roffler-Tarlov S, Schachner M and Freedman LS: Multiple neurotransmitter synthesis by human neuroblastoma cell lines and clones. Cancer Res 38: 3751-3757, 1978.

21. Matthay KK: Neuroblastoma: biology and therapy. Oncology (Williston Park) 11: 1857-66; discussion 1869-72, 1875, 1997.

22. Alán L, Špaček T,Zelenka J, Tauber J, Berková Z,Zacharovová K, Saudek F and Ježek P: Assessment of mitochondrial DNA as an indicator of islet quality: An example in Goto Kakizaki rats. Transplant Proc 43: 3281-3284, 2011.

23. Karess RE, Hayward WS and Hanafusa H: Cellular information in the genome of recovered avian sarcoma virus directs the synthesis of transforming protein. Proc Natl Acad Sci USA 76: 3154-3158, 1979

24. Morton RE and Evans TA: Modification of the bicinchoninic acid protein assay to eliminate lipid interference in determining lipoprotein protein content. Anal Biochem 204: 332-334, 1992.

25. Paulson JR and Laemmli UK: The structure of histone-depleted metaphase chromosomes. Cell 12: 817-828, 1977.
26. Schneider CA, Rasband WS and Eliceiri KW: NIH Image to ImageJ: 25 years of image analysis. Nat Methods 9: 671-675, 2012.

27. Scaduto RC Jr and Grotyohann LW: Measurement of mitochondrial membrane potential using fluorescent rhodamine derivatives. Biophys J 76: 469-477, 1999.

28. Stockwin LH, Yu SX, Borgel S, Hancock C, Wolfe TL, Phillips LR, Hollingshead MG and Newton DL: Sodium dichloroacetate selectively targets cells with defects in the mitochondrial ETC. Int J Cancer 127: 2510-2519, 2010.

29. Cuervo AM, Stefanis L, Fredenburg R, Lansbury PT and Sulzer D: Impaired degradation of mutant alpha-synuclein by chaperone-mediated autophagy. Science 305: 1292-1295, 2004.

30. Maclean KH, Dorsey FC, Cleveland JL and Kastan MB Targeting lysosomal degradation induces p53-dependent cell death and prevents cancer in mouse models of lymphomagenesis. J Clin Invest 118: 79-88, 2008.

31. Maes H, Kuchnio A, Peric A, Moens S, Nys K, De Bock K, Quaegebeur A, Schoors S, Georgiadou M, Wouters J, et al: Tumor vessel normalization by chloroquine independent of autophagy. Cancer Cell 26: 190-206, 2014.

32. Stacpoole PW: The dichloroacetate dilemma: Environmental hazard versus therapeutic goldmine - both or neither? Environ Health Perspect 119: 155-158, 2011.

33. Kankotia S and Stacpoole PW: Dichloroacetate and cancer: New home for an orphan drug? Biochim Biophys Acta 1846: 617-629, 2014.

34. Gong F, Peng X, Sang Y, Qiu M, Luo C, He Z, Zhao X and Tong A: Dichloroacetate induces protective autophagy in LoVo cells: Involvement of cathepsin D/thioredoxin-like protein 1 and Akt-mTOR-mediated signaling. Cell Death Dis 4: e913, 2013

35. Cheng P, Ni Z, Dai X, Wang B, Ding W, Rae Smith A, Xu L, Wu D, He F and Lian J: The novel BH-3 mimetic apogossypolone induces Beclin-1- and ROS-mediated autophagy in human hepatocellular carcinoma cells. [corrected] Cell Death Dis 4: e489, 2013.

36. Wong JYY, Huggins GS, Debidda M, Munshi NC and De Vivo I: Dichloroacetate induces apoptosis in endometrial cancer cells. Gynecol Oncol 109: 394-402, 2008

37. Vella S, Conti M, Tasso R, Cancedda $\mathrm{R}$ and Pagano $\mathrm{A}$ : Dichloroacetate inhibits neuroblastoma growth by specifically acting against malignant undifferentiated cells. Int J Cancer 130 1484-1493, 2012

38. Cai Q, Lu L, Tian J-H, Zhu Y-B, Qiao H and Sheng Z-H Snapin-regulated late endosomal transport is critical for efficient autophagy-lysosomal function in neurons. Neuron 68: 73-86, 2010.

39. Castino R, Fiorentino I, Cagnin M, Giovia A and Isidoro C: Chelation of lysosomal iron protects dopaminergic SH-SY5Y neuroblastoma cells from hydrogen peroxide toxicity by precluding autophagy and Akt dephosphorylation. Toxicol Sci 123: 523-541, 2011

40. Michiorri S, Gelmetti V, Giarda E, Lombardi F, Romano F, Marongiu R, Nerini-Molteni S, Sale P, Vago R, Arena G, et al: The Parkinson-associated protein PINK1 interacts with Beclin1 and promotes autophagy. Cell Death Differ 17: 962-974, 2010.

41. Youle RJ and Narendra DP: Mechanisms of mitophagy. Nat Rev Mol Cell Biol 12: 9-14, 2011.

42. Nakahara K, Ueda M, Yamada K, Koide T, Yoshimochi G, Funayama M, Kim JH, Yamakawa S, Mori A, Misumi Y, et al: Juvenile-onset Parkinsonism with digenic parkin and PINK1 mutations treated with subthalamic nucleus stimulation at 45 years after disease onset. J Neurol Sci 345: 276-277, 2014.

43. Scarffe LA, Stevens DA, Dawson VL and Dawson TM: Parkin and PINK1: Much more than mitophagy. Trends Neurosci 37: 315-324, 2014.

44. Exner N, Treske B, Paquet D, Holmström K, Schiesling C Gispert S, Carballo-Carbajal I, Berg D, Hoepken HH, Gasser T, et al: Loss-of-function of human PINK1 results in mitochondrial pathology and can be rescued by parkin. J Neurosci 27 : 12413-12418, 2007.

45. Dagda RK, Cherra SJ III, Kulich SM, Tandon A, Park D and Chu CT: Loss of PINK1 function promotes mitophagy through effects on oxidative stress and mitochondrial fission. J Biol Chem 284: 13843-13855, 2009

46. Twig G, Elorza A, Molina AJ, Mohamed H, Wikstrom JD, Walzer G, Stiles L, Haigh SE, Katz S, Las G, et al: Fission and selective fusion govern mitochondrial segregation and elimination by autophagy. EMBO J 27: 433-446, 2008. 
47. Santin G, Piccolini VM, Barni S, Veneroni P, Giansanti V, Dal Bo V, Bernocchi G and Bottone MG: Mitochondrial fusion: A mechanism of cisplatin-induced resistance in neuroblastoma cells? Neurotoxicology 34: 51-60, 2013

48. Cipolat S, Martins de Brito O, Dal Zilio B and Scorrano L: OPA1 requires mitofusin 1 to promote mitochondrial fusion. Proc Natl Acad Sci USA 101: 15927-15932, 2004.

49. Zorzano A, Liesa M, Sebastián D, Segalés J and Palacín M: Mitochondrial fusion proteins: Dual regulators of morphology and metabolism. Semin Cell Dev Biol 21: 566-574, 2010.

50. Bertholet AM, Millet AME, Guillermin O, Daloyau M, Davezac N, Miquel M-C and Belenguer P: OPA1 loss of function affects in vitro neuronal maturation. Brain 136: 1518-1533, 2013.

51. Duvezin-Caubet S, Jagasia R, Wagener J, Hofmann S, Trifunovic A, Hansson A, Chomyn A, Bauer MF, Attardi G, Larsson NG, et al: Proteolytic processing of OPA1 links mitochondrial dysfunction to alterations in mitochondrial morphology. J Biol Chem 281: 37972-37979, 2006.
52. Kopek BG, Shtengel G, Grimm JB, Clayton DA and Hess HF: Correlative photoactivated localization and scanning electron microscopy. PLoS One 8: e77209, 2013.

53. Schaffer S, Gruber J, Ng LF, Fong S, Wong YT, Tang SY and Halliwell B: The effect of dichloroacetate on health- and lifespan in C. elegans. Biogerontology 12: 195-209, 2011.

54. Gandhi S, Muqit MMK, Stanyer L, Healy DG, Abou-Sleiman PM, Hargreaves I, Heales S, Ganguly M, Parsons L, Lees AJ, et al: PINK1 protein in normal human brain and Parkinson's disease. Brain 129: 1720-1731, 2006. 